\title{
High-Resolution, High-Accuracy Spectroscopy of Trapped Ions*
}

\author{
D. J. Berkeland*, J. D. Miller ${ }^{\dagger}$, F. C. Cruz ${ }^{\ddagger}$, B. C. Young, \\ R. J. Rafac, X.-P. Huang ${ }^{\S}$, W. M. Itano, J. C. Bergquist and \\ D. J. Wineland
}

National Institute of Standards and Technology (NIST), 325 Broadway, Boulder Colorado 80303

\begin{abstract}
Microwave spectroscopy using trapped and cooled ions can achieve precision and accuracy comparable to the best cesium frequency standards. We discuss standards based on ${ }^{199} \mathrm{Hg}^{+}$ions trapped in linear Paul traps: the Jet Propulsion Laboratory (JPL) standard, which uses up to $10^{7}$ atoms confined near the trap axis, and the recently evaluated NIST standard, which uses approximately ten ions laser cooled and crystalized on the trap axis. We consider future directions in trapped ion frequency standard work, including the use of entangled states for achieving higher precision, and progress on trapped ion optical frequency standards. Finally, we discuss scientific and technical applications of extremely stable frequency standards.
\end{abstract}

\section{INTRODUCTION}

Precise and accurate atomic spectroscopy can rigorously test theories of atomic structure, quantum electrodynamics [1] and other fundamental physics [2,3], determine fundamental constants [4], and provide time and frequency standards [3]. Precise and accurate spectroscopy has two basic requirements. First, the measurement must reach the desired precision in a reasonable averaging time. This requires a good signal to noise ratio and a narrow transition. Second, systematic frequency shifts and broadening mechanisms of the atomic transition being studied must be either very small, or stable and very well measured.

As seen at this conference, many experiments with cooled and trapped ions and with laser-cooled neutral atoms can satisfy these requirements. Here we limit

*) Work performed at NIST is supported by the Office for Naval Research and the Army Research Office. Work of U.S. Government; not subject to U.S. copyright.

*) Los Alamos National Laboratory, P23, MS H803, Los Alamos, NM

†) KLA-Tencor Corp., 1701 Directors Blvd., Suite 1000, Austin, TX 78744

‡) Universidade Estadual de Campinas, UNICAMP-IFGW-DEQ, CP.6165 Campinas, SP, 13083970, Brazil

§) Colorado MED-Tech-RELA, Inc., 6175 Longbow Dr., Boulder, CO 80301 
our discussion to precision experiments using trapped and cooled ions [5]. The systematic shifts of atomic transition frequencies in these systems can be small and well-characterized. For example, Stark shifts are small since $\langle\mathbf{E}\rangle=0$ and $\left\langle E^{2}\right\rangle$ is small when the ions are cold. The magnetic field can be small and easy to characterize because the ions occupy a small volume. The corresponding Zeeman shift is typically small because usually transitions with only a second-order field dependence are used. Because background gas pressure can be negligible, collisional shifts and broadening can be small. The ions can be cooled using either buffer-gas cooling or laser cooling [6], reducing Doppler shifts. The statistical precision can be high if large numbers of ions are stored, or if the shot noise limit is reached. Finally, free precession times of several minutes have been reported $[7,8]$, giving extremely narrow transition linewidths.

The statistical precision of a frequency standard can be predicted quantitatively for various cases. For example, if an atomic transition is probed using the Ramsey technique [9] and the measurement precision is limited only by quantum fluctuations in the atomic state populations [10], the fractional precision of the frequency measurement is given by [11]

$$
\frac{\Delta \omega_{\text {measured }}}{\omega_{0}} \equiv \sigma_{y}(\tau)=\frac{1}{\omega_{0} \sqrt{N T_{R}}} \tau^{-1 / 2} .
$$

Here, $\omega_{0}$ is the transition angular frequency, $\Delta \omega_{\text {measured }}$ is the precision with which the frequency is measured, and the Allan deviation $\sigma_{y}(\tau)$ is related to the fractional frequency instability. $N$ is the number of atoms used, $T_{R}$ is the free precession time between the two $\pi / 2$ Rabi pulses, and $\tau\left(>T_{R}\right)$ is the averaging time of the measurement. Ideally, $N, T_{R}$, and $\tau$ are large, although various experimental constraints may limit these values.

Table 1 compares these parameters for two types of microwave frequency standards-those based on ions confined in an rf Paul trap and those based on a pulsed fountain of cesium atoms [12]. The number $N$ of atoms used in the trapped ion standards is limited in part by the second-order Doppler shift due to micromotion. This motion is driven by the trap's rf electromagnetic fields, and becomes greater as the Coulomb repulsion between the ions forces them further from the field nodal point or line [13]. Thus there is a trade-off between using very large $N$ but with a substantial Doppler shift, or a negligible Doppler shift but smaller $N$. In the Cs fountain standard, $N$ is limited in part by collisional shifts. For laser-cooled trapped ion standards, $T_{R}$ is limited by the time the ions remain cold in the absence of cooling radiation. In a fountain standard it is limited to about $1 \mathrm{~s}$ by the maximum practical height the atoms can be tossed (about one meter). Finally, the averaging time $\tau$ is not fundamentally limited in either type of standard.

Ions in Penning traps have been used previously to realize the first laser-cooled frequency standard $[7,16]$. An important limitation to accuracy in those experiments was the uncertainty in the second-order Doppler shift due to the overall rotational motion of the ion cloud. This motion can now be precisely controlled 
TABLE 1. Example parameters for trapped ion and cesium fountain microwave frequency standards.

\begin{tabular}{ccc}
\hline Parameter & Ions & Cs \\
\hline$\omega_{0} /(2 \pi)$ & $40.5 \mathrm{GHz}\left({ }^{199} \mathrm{Hg}^{+}\right)$ & $9.2 \mathrm{GHz}$ \\
$N$ & $\approx 10[14]$ to $10^{7}[15]$ & $10^{4}$ to $10^{6}$ \\
$T_{R}$ & Up to $600 \mathrm{~s}[7,8]$ & $\sim 1 \mathrm{~s}$ \\
\hline
\end{tabular}

[17], and it should therefore be possible to realize an of or microwave frequency standard with accuracy comparable to what is possible with Paul traps [18]. To date, the highest accuracies and stabilities have been obtained in linear Paul traps; therefore, we highlight, this work below.

Figure 1 shows a schematic diagram of a linear Paul trap [19-21]. In this trap, the ions are confined axially by the two cylindrical sections (endcaps) held at static potential $U_{0}$. The shape of these endcaps is not critical, and axial confinement can be produced by thin conducting rods located on the trap axis at both ends of the trap [15], by small rings [20] or by segmented electrodes [21]. Two of the long, thin rods of Fig. 1 are held at ground potential, while the other two are held at an rf potential. This gives an oscillating electric potential that traps the ions in a radial quadratic pseudopotential [22]. The advantage of the linear trap is that many ions can be confined near or on the electric field nodal line, where Doppler shifts from micromotion are minimized.

\section{MICROWAVE SPECTROSCOPY}

Microwave spectroscopy with clouds of trapped ions has been used for many years in atomic structure measurements [23]. Some recent experiments report measurements of $g$ factors [24] and hyperfine constants [25-29]. Because ion traps can use small samples, they are well-suited for measurements of hyperfine anomalies using

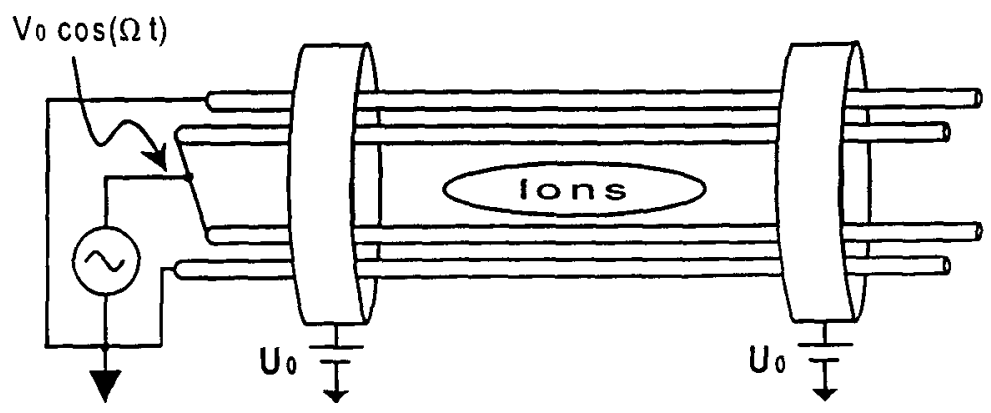

FIGURE 1. Schematic diagram of a linear Paul trap. 


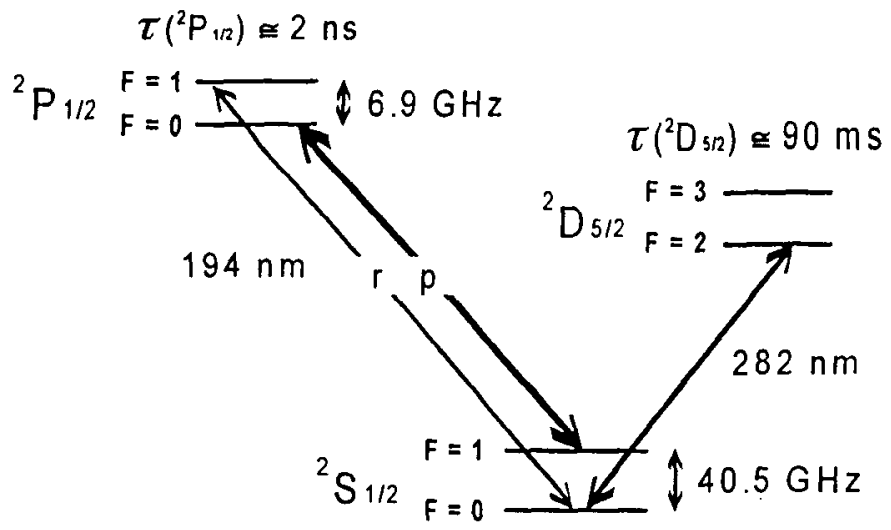

FIGURE 2. Partial energy level diagram of ${ }^{199} \mathrm{Hg}^{+}$.

different isotopes, some of which are radioactive $[25,26,29]$.

Taking advantage of the precision and accuracy offered by the ground state hyperfine transition of trapped ions, several groups are working on atomic frequency standards based on ${ }^{113} \mathrm{Cd}^{+}\left(\omega_{0}=2 \pi \times 15.2 \mathrm{GHz}\right)[27,30]$ and ${ }^{171} \mathrm{Yb}^{+}$ $\left(\omega_{0}=2 \pi \times 12.6 \mathrm{GHz}\right) \quad[20,31-33]$. Here we describe microwave frequency standards based on trapped ${ }^{199} \mathrm{Hg}^{+}$ions $[14,15,34]$. Figure 2 shows a partial energy level diagram of this atom. The electric dipole transitions at $194 \mathrm{~nm}$ are used for state preparation and detection, and can be used for laser cooling. The ground state hyperfine splitting frequency is $2 \pi \times 40.5 \mathrm{GHz}$, the highest of routinely trapped ions. The $\Delta m_{F}=0$ hyperfine transition depends only quadratically on the magnetic field, when the field is near zero.

At JPL, much work has been done with clouds of ${ }^{199} \mathrm{Hg}^{+}$ions in a linear Paul trap $[15,34]$. The methods and performance of the Commonwealth Scientific and Industrial Research Organization (CSIRO) experiments on ${ }^{171} \mathrm{Yb}^{+}$are similar [20]. The four trap rods of the JPL linear ion trap standard (LITS) in reference [15] are evenly spaced on a circle of $1 \mathrm{~cm}$ radius, with two endcap rods on the trap axis $7.5 \mathrm{~cm}$ apart. This trap confines clouds of up to $10^{7}$ ions, which are cooled with a helium buffer gas. States are prepared and detected with a ${ }^{202} \mathrm{Hg}$ lamp, which emits broad-line radiation that partially overlaps the resonances of the two ${ }^{2} S_{1 / 2}, F=1 \rightarrow{ }^{2} P_{1 / 2}, F=0,1$ transitions. Because the ${ }^{2} P_{1 / 2}, F=1$ state can decay to the ${ }^{2} S_{1 / 2}, F=0$ state, illuminating the ions with the lamp radiation pumps them into the ${ }^{2} S_{1 / 2} F=0$ state in preparation for the Ramsey interrogation. At the end of the Ramsey interrogation, the lamp radiation is returned to the ions, and the detected fluorescence indicates how many ions are in the ${ }^{2} S_{1 / 2} F=1$ state. Because the ions scatter only a few photons before optically pumping out. of the ${ }^{2} S_{1 / 2} F=1$ state, and because only a small fraction of the scattered photons are detected, the state detection efficiency is much less than unity, so the state measurements are not quantum noise limited and Eq. (1) is not applicable. 
However, because $N$ is large, this device is very stable. With a Ramsey time $T_{R}$ of $8 \mathrm{~s}$, LITS has recently demonstrated an instability of $\sigma_{y}(\tau)=3 \times 10^{-14} \tau^{-1 / 2}[35]$. At CSIRO, a similar standard using clouds of laser-cooled ${ }^{171} \mathrm{Yb}^{+}$ions in a linear trap has $\sigma_{y}(\tau)=4.7 \times 10^{-14} \tau^{-1 / 2}[20]$.

Although these standards are very stable, the second-order Doppler shift from the ion motion is substantial (about $9 \times 10^{-13}$ ). At NIST, the goal is to develop a frequency standard that is both stable and accurate. We use a linear trap such as that shown in Fig. 1, with the endcaps approximately $4 \mathrm{~mm}$ apart and the $0.2 \mathrm{~mm}$ radius rods on a $0.64 \mathrm{~mm}$ radius [36]. Approximately ten ions are used, and they are laser cooled so that they crystallize along the nodal line of the $\mathrm{rf}$ electric field, near the trap axis. Using a small number of ions sacrifices precision according to Eq. (1), but greatly reduces Doppler shifts. Groups at CSIRO [37] and the Communications Research Laboratory (CRL) [38] have also crystalized lasercooled ions in linear Paul traps in order to improve the accuracy of their frequency standards.

The NIST standard has other advantages. The trap is enclosed in a copper container that forms the bottom of a liquid helium reservoir [36]. Because of the cryogenic environment, the pressure of background neutral mercury atoms is negligible. This is critical because the background mercury pressure leads to ion loss, presumably due to dimer formation. This ion loss limits the storage time of trapped ions in a room temperature trap to about ten minutes. At $4 \mathrm{~K}$, the ions can be trapped for days at a time without loss. Also, in the $4 \mathrm{~K}$ environment the pressure of all other background gases is negligible, with the possible exception of helium. This greatly reduces collisional shifts and heating from collisions. Additionally, the black body shift, which is already over two orders of magnitude smaller than in cesium at room temperature [39], is dramatically reduced. To ensure that the ions are on the nodal line, we minimize the micromotion observed in three non-coplanar directions [13]. Because the if electric field at the site of the ions in minimized, the rf heating while the cooling lasers are off during the free precession time $T_{R}$ is minimized. Finally, laser cooling (instead of buffer gas cooling) significantly reduces Doppler shifts; we have measured the second-order Doppler shift to be less than $3 \times 10^{-17}[14]$.

The steps in operating the frequency standard are as follows. We Doppler cool the ions by using radiation from a primary laser whose frequency is slightly reddetuned from transition $p$ (see Fig. 2). Although this is a cycling transition, the laser weakly couples the $\mathrm{S}_{1 / 2}, F=1$ state to the $\mathrm{P}_{1 / 2}, F=1$ state, which decays to the $S_{1 / 2}, F=0$ state. To optically pump the ions out of this state, we overlap a less intense repumping laser beam with the primary laser beam to drive the $r$ transition. After a Doppler cooling period of 200 to $300 \mathrm{~ms}$, we pump the ions into the $\mathrm{S}_{1 / 2}, F=0$ state with high efficiency by applying only the primary laser for $10 \mathrm{~ms}$. Then we drive the microwave transition using the Ramsey method, with a free precession time $T_{R}$ of 2 to $100 \mathrm{~s}$. After this, to determine the ensemble average of the $S_{1 / 2}, F=1$ state populations, we again apply only the primary laser for about $10 \mathrm{~ms}$. If an ion is found to be in the $F=1$ state, it will scatter 


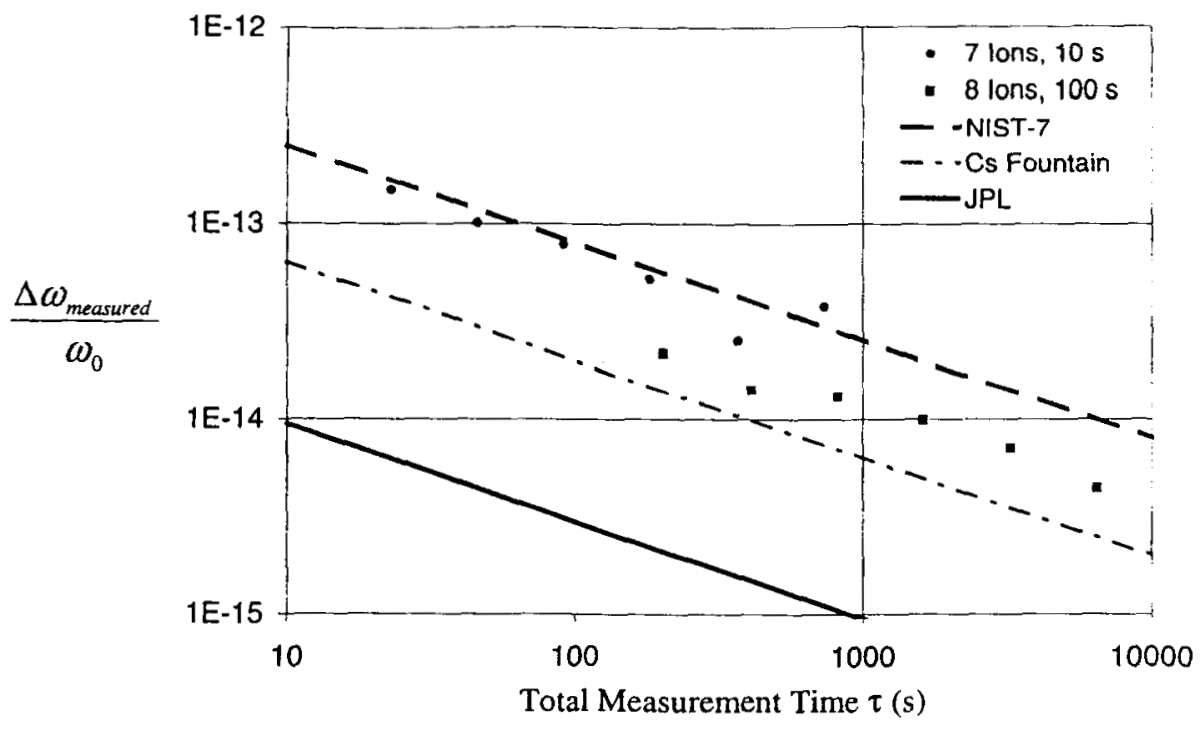

FIGURE 3. Stabilities of several standards.

approximately 10,000 photons before being optically pumped into the $\mathrm{S}_{1 / 2}, F=0$ state. Otherwise, the atoms scatter only a few photons in the same time interval, due to the off-resonant $\mathrm{S}_{1 / 2}, F=0$ to $\mathrm{P}_{1 / 2}, F=1$ transition. Our detection signal is the observed fluorescence from all of the ions. Repeating this measurement process and scanning the microwave frequency produces a Ramsey fringe pattern. We lock the frequency to the central Ramsey fringe by stepping the frequency from one side of the fringe to the other, while a digital servo works to keep the detection signal constant at each step.

Figure 3 shows the instability $\sigma_{y}(\tau)$ of the microwave oscillator when it is locked to the central Ramsey fringe with $T_{R}=10 \mathrm{~s}$ and $T_{R}=100 \mathrm{~s}$. For $T_{R}=100 \mathrm{~s}$, the instability is $\sigma_{y}(\tau)=3.3 \times 10^{-13} \tau^{-1 / 2}$. The measured instability of the standard is consistently twice that expected from Eq. (1), due to fluctuations of the laser intensity at the site of the ions. The figure also shows the stability of the cesium beam standard NIST-7 $\left(8 \times 10^{-13} \quad \tau^{-1 / 2}\right)[40]$ and the Paris cesium fountain standard $\left(2 \times 10^{-13} \tau^{-1 / 2}\right)$ [12]. The instabilities of these three standards are comparable, while that of the $\mathrm{JPL}{ }^{199} \mathrm{Hg}^{+}$standard is significantly better $\left(\sigma_{y}(\tau)=3 \times 10^{-14} \tau^{-1 / 2}\right)$.

The accuracy of the JPL standard is limited by the second-order Doppler shift to around $10^{-13}$ [41]. The other standards in Fig. 3 have significantly greater accuracy, and the accuracies of these standards are comparable. The fractional accuracy of NIST-7 is $5 \times 10^{-15}$, limited by the distributed cavity phase shift [42]. This high accuracy corresponds to splitting the central Ramsey fringe to about a part in $10^{6}$. A natural next step toward a more accurate frequency standard is to reduce the 
linewidth. The cesium fountain frequency standard does this by increasing the Ramsey free precession time $T_{R}$. In this standard, the accuracy is currently limited by black body and collisional shifts to $1.4 \times 10^{-15}[43]$. The accuracy of the NIST ${ }^{199} \mathrm{Hg}^{+}$standard is $3.4 \times 10^{-15}$, and is limited by uncertainties in the Zeeman shift [14]. The dominant shift is caused by asymmetries in currents running through the trap electrodes, which cause a net rf magnetic field at the site of the ions.

The trapped ion and cesium fountain standard are both emerging technologies that promise even higher accuracy. At. NIST we have constructed a new, smaller trap, which we will run with lower trap drive frequency $\Omega$. Less rf potential is needed for the same radial confinement in the trap, reducing the currents in the trap rods and, from scaling arguments, the associated magnetic field at the site of the ions. Also, we have improved our magnetic shielding to reduce fluctuations in the ambient magnetic field. We expect that the next generation of this standard will be accurate to approximately $10^{-16}$.

\section{FUTURE DIRECTIONS}

One way to reduce the instability $\sigma_{y}(\tau)$ of a standard is to increase the transition frequency $\omega_{0}$. Therefore, many groups are working on optical frequency standards. The laser-cooled stored ion efforts include trapped ${ }^{172} \mathrm{Yb}^{+}$at $411 \mathrm{~nm}$ [44] and $3.43 \mu \mathrm{m}[45],{ }^{171} \mathrm{Yb}^{+}$at $435 \mathrm{~nm}[46],{ }^{88} \mathrm{Sr}^{+}$at $674 \mathrm{~nm}[47,48],{ }^{138} \mathrm{Ba}^{+}$at $12.5 \mu \mathrm{m}$ [49], ${ }^{40} \mathrm{Ca}^{+}$at $729 \mathrm{~nm}[50-52],{ }^{115} \mathrm{In}^{+}$at $237 \mathrm{~nm}[53,54]$, and ${ }^{199} \mathrm{Hg}^{+}$at $282 \mathrm{~nm} \mathrm{[55].}$

Earlier work at NIST using ${ }^{199} \mathrm{Hg}^{+}$in a room temperature trap produced a narrow transition with structure due to Rabi oscillations [55]. The width of the central feature was about $40 \mathrm{~Hz}$ at $563 \mathrm{~nm}$. Our pursuit of higher resolution and a study of systematic effects was hampered by the limited lifetime of the ion in the room temperature trap. However, we have now built a second cryogenic system that will provide long ion lifetimes and more detailed investigations of this transition. Also, we have recently made substantial improvements in the laser system; the laser linewidth in about a one minute averaging time is now less than one hertz at. $563 \mathrm{~nm}[56]$.

For the absolute frequency of a transition to be determined, $\omega_{0}$ must be compared to an accepted frequency standard. We are now comparing the frequency of the $282 \mathrm{~nm}$ transition to a that of the narrow ${ }^{1} S_{0}-{ }^{3} P_{1}$ intercombination line of $\mathrm{Ca}$ at $657 \mathrm{~nm}[57,58]$ by mixing a CO overtone line with the fundamental $563 \mathrm{~nm}$ light [59]. The NIST Ca standard has been compared to the Physikalisch-Technische Bundesanstalt (PTB) Ca standard, which has in turn been compared to the $9.2 \mathrm{GHz}$ line in Cs [60]. In other laboratories, the absolute frequency of the ${ }^{88} \mathrm{Sr}^{+} \mathrm{S}$ to $\mathrm{D}$ transition at $674 \mathrm{~nm}$ has been measured with a frequency chain [48]. Interferometric measurements have also been made on this transition [61] and on the $S$ to $D$ transition at $411 \mathrm{~nm}$ in ${ }^{172} \mathrm{Yb}^{+}$[44], with frequency chain measurements in progress [62].

Another way to reduce $\sigma_{y}(\tau)$ is by using entangled states $[63,64]$. Consider 
an atomic system with two states labeled $\uparrow$ and $\downarrow$, separated by frequency $\omega_{0}$. We use the spin $1 / 2$ analog for these two states [65], so the total angular momentum for $N$ ions is given by $\mathrm{J}=\sum_{i=1}^{N} \mathrm{~S}_{\mathbf{i}}$, where $\mathrm{S}_{\mathbf{i}}$ is the spin of the ith atom $\left(S_{i}=1 / 2\right)$. For uncorrelated atoms, the Ramsey spectrum is given by $\left\langle J_{z}\right\rangle=N \cos \left(\left(\omega_{0}-\omega\right) T_{R}\right)$. The best possible uncertainty in the measured value of $\omega_{0}$ is the shot noise limit of $\Delta \omega_{0}=1 / \sqrt{N T_{R} \tau}$ [10]. This uncertainty can be reduced if we use an entangled state in the following way. Suppose that the $\mathrm{N}$-atom state at the beginning of the free precession time is given by

$$
\left(e^{\frac{-i N \omega_{0} t}{2}}\left|\uparrow_{1} \uparrow_{2} \ldots \uparrow_{N}>+e^{\frac{+i N \omega_{0} t}{2}}\right| \downarrow_{1} \downarrow_{2} \ldots \downarrow_{N}>\right) / \sqrt{2} .
$$

After the free precession time, a $\pi / 2$ Rabi pulse is applied as in usual Ramsey spectroscopy. The signal is obtained by measuring an operator that is the product of the $z$-components of the Pauli spin matrices, $O=\prod_{i=1}^{N}\left(\sigma_{z}\right)_{i}$. This gives a signal $\langle O\rangle=(-1)^{N} \cos \left(N\left(\omega_{0}-\omega\right) T_{R}\right)$, where $\omega$ is the frequency of the applied radiation. The uncertainty in $\omega_{0}$ is now given by the exact Heisenberg limit $\Delta \omega_{0}=$ $1 / N \sqrt{T \tau}$ [63]. This method results in a factor of $N$ decrease in the averaging time $\tau$ required to achieve a given precision. Although similar precision gains can be made by increasing $N$, the optimal value of $N$ in high accuracy ion trap standards might be limited by other experimental constraints, thereby making entangled state spectroscopy advantageous.

\section{APPLICATIONS}

Improved frequency standards benefit communications and navigation $[66,67]$ and help in determining some fundamental physical constants [68]. Another possible application of improved frequency standards is in detecting the stochastic background of gravitational radiation $[69,70]$. This background is similar to the observed cosmic microwave background at $4 \mathrm{~K}$, but has not been detected. Estimates for the density $\rho$ of these gravitational waves vary by many orders of magnitude and are extremely model dependent. A limit on $\rho$ may be determined by timing the bursts of light emitted by pulsars, which are more stable for long times than the best clocks on earth [71]. If gravitational radiation present at the pulsar location is not correlated with the gravitational radiation present at earth, clocks at the two locations will become decorrelated after some time $\tau_{d}$, and the measured instability $\sigma_{y}(\tau)$ of the pulsar bursts will begin to increase with averaging time $\tau>\tau_{d}$. For example, using the estimates from [72], timing measurements from the pulsar J1713+0747 should destabilize at $\tau=\tau_{d} \sim 2 \mathrm{y}$ and $\sigma_{y}\left(\tau_{d}\right) \sim 10^{-14}$ [73]. However, because the current global time scale is stable at only the $10^{-14}$ level on a time scale of about one year, any observed destabilization could also be from the long-term drifts in the terrestrial time scale. Better clocks are thus required to verify or reject these theories.

Another scientific application of better frequency standards is the laboratory measurement of possible changes in fundamental constants, such 
as the fine structure constant $\alpha$. Nonlaboratory measurements include estimates from $\mathrm{Sm}$ isotope distributions at the Oklo mine, giving $-6.7 \times 10^{-17} /$ year $<\dot{\alpha} / \alpha<5.0 \times 10^{-17} /$ year [74]. However, this measurement assumes a linear change in $\alpha$ over time and is model dependent. Laboratory measurements can in principle detect nonlinear changes in $\alpha$ over a relatively short. time. Because the hyperfine splitting frequency depends nonlinearly on the nuclear charge $Z$ and on $\alpha$, comparing the frequency of the hyperfine constant in hydrogen $A_{H}$ to that of another alkali $A_{\text {Alkali }}$ gives $\dot{\alpha} / \alpha$ according to [75]

$$
\frac{d}{d t} \ln \frac{A_{\text {Alkali }}}{A_{H}}=\alpha \frac{d}{d \alpha} \ln \left(F_{\text {rel }}(\alpha Z)\right)\left(\frac{1}{\alpha} \frac{d \alpha}{d t}\right)
$$

where $F_{\text {rel }}(\alpha Z)$ is the Casimir correction factor [76]. Recently, the hyperfine structure frequency of ${ }^{199} \mathrm{Hg}^{+}$has been compared to that of hydrogen for 140 days to obtain $|\alpha / \alpha| \leq 3.7 \times 10^{-14} /$ year [75]. Optical atomic transition measurements have also been proposed for detecting changes in $\alpha$ [77]. Clearly, any improvement. in the precision of laboratory frequency standards would tighten the limits on $\dot{\alpha} / \alpha$.

\section{SUMMARY}

Trapped and cooled ions are particularly suitable systems for precise and accurate spectroscopy. Frequency standards using trapped ions have stabilities and accuracies that are comparable to those of the best of Cs standards and are expected to continue to improve. Some directions toward improving trapped ion frequency standards use optical transitions and entangled states. In addition to improving technological applications, better frequency standards can test fundamental physics.

\section{ACKNOWLEDGMENTS}

We thank Lute Maleki, Travis Mitchell, John Prestage, Don Sullivan and Matt. Young for valuable comments on this manuscript.

\section{REFERENCES}

1. Kinoshita, T., editor, Quantum Electrodynamics, World Scientific, Singapore, 1990.

2. Maleki, L., editor, Proceedings of the Workshop on the Scientific Applications of Clocks in Space, 1997, JPL Publication 97-15.

3. Bergquist, J., editor, Proceedings of the 5th Symposium on Frequency Standards and Metrology, Singapore, 1996, World Scientific.

4. Petley, B. W., The Fundamental Physical Constants and the Frontier of Measurement, A. Higler, Bristol, England, 1988.

5. Fisk, P. T. H., Rep. Prog. Phys. 60, 761 (1997). 
6. Itano, W. M., Bergquist, J. C., Bollinger, J. J., and Wineland, D. J., Phys. Rev. ST 59, 106 (1995).

7. Bollinger, J. J., Heinzen, D. J., Itano, W. M., Gilbert, S. L., and Wineland, D. J., IEEE Trans. Instrum. Meas. 40, 126 (1991).

8. Fisk, P. T. H., Sellars, M. J., Lawn, M. A., Coles, C., Mann, A. G., and Blair, D. G., IEEE Trans. Instrum. Meas. 44, 113 (1995).

9. Ramsey, N. F., Molecular Beams, Oxford University Press, London, 1956.

10. Itano, W. M., Bergquist, J. C., Bollinger, J. J., Gilligan, J. M., Heinzen, D. J., Moore, F. L., Raizen, M. G., and Wineland, D. J., Phys. Rev. A 47, 3554 (1993).

11. Wineland, D. J., Itano, W. M., Bergquist, J. C., Bollinger, J. J., Dietrich, F., and Gilbert, S. L., "High Accuracy Spectroscopy of Stored Ions," in Proceedings of the 4th Symposium on Frequency Standards, edited by Demarchi, A., pages 71-76, Heidelberg, 1989, Springer-Verlag.

12. Clairon, A., Ghezali, S., Santarelli, G., Laurent, P., Lea, S., Bahoura, M., Simon, E., Weyers, S., and Szymaniec, K., "Preliminary Accuracy Evaluation of a Cesium Fountain Frequency Standard," in Bergquist [3], pages 49-59.

13. Berkeland, D. J., Miller, J. D., Bergquist, J. C., Itano, W. M., and Wineland, D. J., J. Appl. Phys. 83, 5025 (1998).

14. Berkeland, D. J., Miller, J. D., Bergquist, J. C., Itano, W. M., and Wineland, D. J., Phys. Rev. Lett. 80, 2089 (1998).

15. Tjoelker, R., Prestage, J. D., and Maleki, L., "Record Frequency Stability with Mercury in a Linear Ion Trap," in Bergquist [3], pages 33-38.

16. Bollinger, J. J., Prestage, J. D., Itano, W. M., and Wineland, D. J., Phys. Rev. Lett. 54, 1000 (1985).

17. Huang, X.-P., Bollinger, J. J., Mitchell, T. B., and Itano, W. M., Phys. Rev. Lett. 80, 73 (1998).

18. Tan, J. N., Bollinger, J. J., and Wineland, D. J., IEEE Trans. Instrum. Meas. 44, 144 (1995).

19. Prestage, J. D., Dick, G. J., and Maleki, L., J. Appl. Phys. 66, 1013 (1989).

20. Fisk, P. T. H., Sellars, M. J., Lawn, M. A., and Coles, C., IEEE Trans, Ultrason., Ferroel. and Freq. Control 44, 344 (1997).

21. Raizen, M. G., Gilligan, J. M., Bergquist, J. C., Itano, W. M., and Wineland, D. J., J. Mod. Optics 39, 233 (1992).

22. Paul, W., Rev. Mod. Phys. 62, 531 (1990).

23. Werth, G., Hyperfine Interactions 99, 3 (1996).

24. Knöll, K. H., Marx, G., Hübner, K., Schweikert, F., Stahl, S., Weber, C., and Werth, G., Phys. Rev. A 54, 1199 (1996).

25. Enders, K., Stachowska, E., Marx, G., Zölch, C., Georg, U., Dembczynski, J., and Werth, G., Phys. Rev. A 56, 265 (1997).

26. Wada, M., Okada, K., Wang, H., Enders, K., Kurth, F., Nakamura, T., Fujitaka, S., Tanaka, J., Kawakami, H., Ohtoni, S., and Katayama, I., Nucl. Phys. A 626, 365c (1997).

27. Tanaka, U., Imajo, H., Hayasaka, K., Ohmukai, R., Watanabe, M., and Urabe, S., Phys. Rev. A 53, 3982 (1996).

28. Arbes, F., Benzing, M., Gudjons, T., Kurth, F., and Werth, G., Z. Phys. D 31, 27 
(1994).

29. Sunaoshi, H., Fukashiro, Y., Furukawa, M., Yamauchi, M., Hayashibe, S., Shinozuka, T., Fujioka, M., Satoh, I., Wada, M., and Matsuki, S., Hyperfine Interactions 78, 241 (1993).

30. Matsubara, K., Tanaka, U., Imajo, H., Hayasaka, K., Ohmukai, R., Watanabe, M., and Urabe, S., Appl. Phys. B 67, 1 (1998).

31. Tamm, C., Schnier, D., and Bauch, A., Appl. Phys. B 60, 19 (1995).

32. Enders, V., Courteille, P., Huesmann, R., Ma, L. S., Neuhauser, W., Blatt, R., and Toschek, P. E., Europhys. Lett. 24, 325 (1993).

33. Seidel, D. J., Williams, A., Berends, R. W., and Maleki, L., "The Development of the Ytterbium Ion Frequency Standard," in 1992 IEEE Frequency Control Symposium, pages 70-75, New York, 1992, IEEE.

34. Prestage, J. D., Tjoelker, R. L., Dick, G. J., and Maleki, L., J. Mod. Optics 39, 221 (1992).

35. Maleki, L., MS 298-100, JPL, 4800 Oak Grove Drive, Pasadena, CA 91109, private communication.

36. Poitzsch, M. E., Bergquist, J. C., Itano, W. M., and Wineland, D. J., Rev. Sci. Instrum. 67, 129 (1996).

37. Fisk, P. T. H., National Measurement Laboratory, CSIRO Division of Telecommunications and Industrial Physics, PO Box 218, Lindfield NSW 2070, Sydney, Australia, private communication.

38. Tanaka, U., Kansai Research Center, Communications Research Laboratory, 588-2, Iwaoka, Nishi-ku, Kobe 651-24, Japan, private communication.

39. Itano, W. M., Lewis, L. L., and Wineland, D. J., Phys. Rev. A 23, 1233 (1982).

40. Lee, W. D., Shirley, J. H., Lowe, J. P., and Drullinger, R. E., IEEE Trans. Instrum. Meas. 44, 120 (1995).

41. Tjoelker, R., Prestage, J. D., and Maleki, L., "Long Term Stability of $\mathrm{Hg}^{+}$Trapped Ion Frequency Standards," in Proceedings of the 1993 IEEE International Frequency Control Symposium, pages 132-138, Piscataway, NJ, 1993, IEEE.

42. Drullinger, R. E., Shirley, J. H., and Lee, W., "NIST-7, the U.S. Primary Frequency Standard: New Evaluation Techniques," in 28th Annual PTTI Application and Planning Meeting, pages 255-264, 1997.

43. Clairon, A., 1998, presented at the 1998 Conference on Precision Electromagnetic Measurements.

44. Taylor, P., Roberts, M., Gateva-Kostove, S. V., Clarke, R. B. M., Barwood, G. P., and Rowley, W. R. C., Phys. Rev. A 56, 2699 (1997).

45. Taylor, P., Roberts, M., Barwood, G. P., and Gill, P., Opt. Lett. 23, 298 (1998).

46. Tamm, C. and Engelke, D., "Optical Frequency Standard Investigations on Trapped ${ }^{171} \mathrm{Yb}$ Ions," in Bergquist [3], pages 283-288.

47. Barwood, G. P., Gill, P., Huang, G., Klein, H. A., and Rowley, W. R. C., Opt. Commun. 151, 50 (1998).

48. Madej, A. A., 1998, these proceedings.

49. Madej, A. A., Siemsen, K. J., Whitford, B. G., Bernard, J. E., and Marmet, L., "Precision Absolute Frequency Measurements with Single Atoms of $\mathrm{Ba}^{+}$and $\mathrm{Sr}^{+}$," in Bergquist [3], pages 165-170. 
50. Urabe, S., Watanabe, M., Imago, H., Hayasaka, K., and Tanaka, U., "Observation of Doppler sidebands of a laser-cooled $\mathrm{Ca}^{+}$ion by using a low temperatureoperated laser diode," in Internation Workshop on Current Topics of Laser Technology, page 55, Tokyo, 1998, Communications Research Laboratory.

51. Knoop, M., Vedel, M., and Vedel, F., J. Phys. (Paris) II 4, 1639 (1994).

52. Plumelle, F., Desaintfuscien, M., and Houssin, M., IEEE Trans. Instrum. Meas. 42 , 462 (1993).

53. Peik, E., Hollemann, G., Abel, J., v. Zanthier, J., and Walther, H., "Single-ion Spectroscopy of Indium: Towards a Group-III Monoion Oscillator," in Bergquist [3], pages $376-379$.

54. Nagourney, W., Burt, E., and Dehmelt, H. G., "Optical Frequency Standard Using Individual Indium Ions," in Bergquist [3], pages 341-346.

55. Bergquist, J. C., Itano, W. M., and Wineland, D. J., "Laser Stabilization to a Single Ion," in Frontier in Laser Spectroscopy, edited by Hänsch, T. and Inguscio, M., pages 359-376, Amsterdam, 1994, North Holland.

56. Young, B. C., Cruz, F. C., and Bergquist, J. C., in preparation.

57. Riehle, F., Schnatz, H., Lipphardt, B., Zinner, G., Kersten, P., and Helmcke, J., "Optical Frequency Standard Based on Laser-cooled Ca Atoms," in Bergquist [3], pages 277-282.

58. Zibrov, A. S., Fox, R. W., Ellingsen, R., Wiemer, C. S., Velichansky, V. L., Tino, G. M., and Hollberg, L., Appl. Phys. B 59, 327 (1994).

59. Frech, B., Wells, J., Hollberg, L., Oates, C., Young, B. C., and Bergquist, J. C., in preparation.

60. Schnatz, H., Lipphardt, B., Helmcke, J., Riehle, R., and Zinner, G., Phys. Rev. Lett. 76, 18 (1996).

61. Barwood, G. P., Edwards, C. S., Gill, P., Huang, G., Klein, H. A., and Rowley, W. R. C., IEEE Trans. Instrum. Meas. 44, 117 (1995).

62. Lea, S. N. and Gill, P., "Proposed Infra-red to Visible Frequency Chain at NPL," in Bergquist [3], pages 507-508.

63. Bollinger, J. J., Itano, W. M., Wineland, D. J., and Heinzen, D. J., Phys. Rev. A 54, R4649 (1996).

64. Wineland, D. J., Monroe, C., Itano, W. M., Leibfried, D., King, B., and Meekhof, D., NIST J. Res. 103, 259 (1998).

65. Feynman, R. P., Vernon, F. L., and Hellwarth, R. W., J. Appl. Phys. 28, 49 (1957).

66. Hellwig, H., IEEE Trans. Ultrason., Ferroel. and Freq. Control 40, 538 (1993).

67. Vig, J. R., IEEE Trans. Ultrason., Ferroel. and Freq. Control 40, 522 (1993).

68. Petley, B. W., Proc. IEEE 79, 1070 (1993).

69. Detweiler, S., Astrophys. J. 234, 1100 (1979).

70. Thorne, K. S., 300 Years of Gravitation, pages 330-458, Cambridge University Press, Cambridge, 1987.

71. Taylor, J. H., Proc. IEEE 79, 1054 (1991).

72. Vachaspati, T. and Vilenkin, A., Phys. Rev. D 31, 3052 (1985).

73. Foster, R. S., Camilo, F., and Wolszczan, A., "High-precision Metrology from Pulsar J1713+0747," in Marcel Grossmann Conference on General Relativity, 1995.

74. Damour, T. and Dyson, F., Nucl. Phys. B 480, 37 (1996). 
75. Prestage, J. D., Tjoelker, R. L., Dick, G. J., and Maleki, L., Phys. Rev. Lett. 74. 3511 (1995).

76. Casimir, H. B. G., On the Interaction between Atomic Nuclei and Electrons, Freeman. San Francisco, 1963, p. 54.

77. Dzuba, V. A., Flambaum, V. V., and Webb, J. K., "Space-time Variation of Physical Constants and Relativistic Corrections in Atoms," 1998, submitted to Phys. Rev. Lett.: preprint physics/9802029 on http://xxx.lanl.gov. 